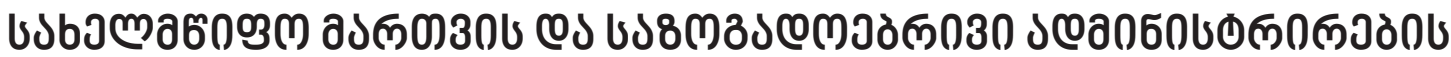

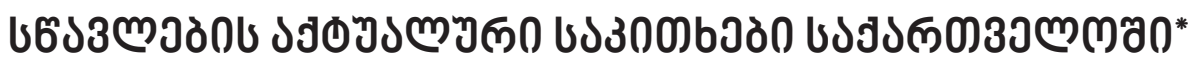

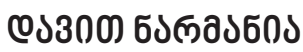

https://doi.org/10.35945/gb.2018.06.009

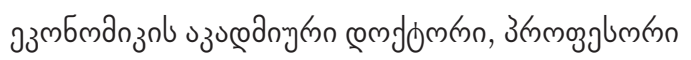

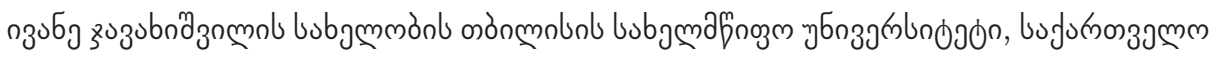

davit.narmania@tsu.ge

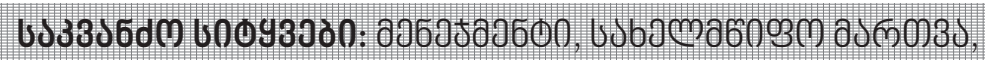

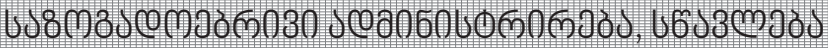

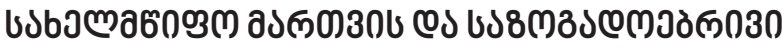

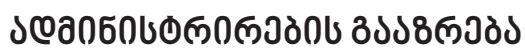

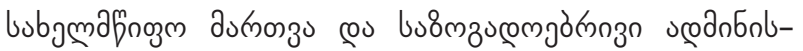

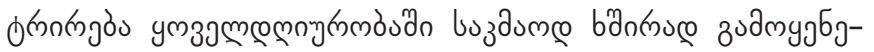
১uçn ogr

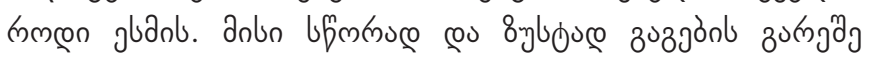

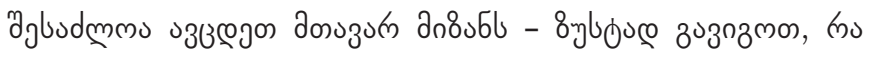

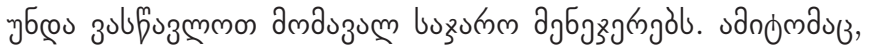

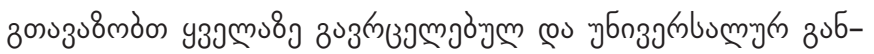

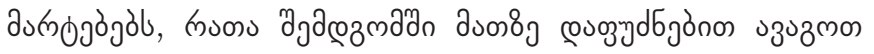

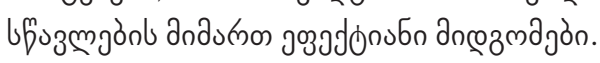

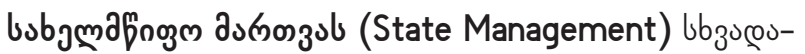
ubzu bnбmbnan zushbnu, mmzmmnzus buzuknm asknonzu (Public Management), buzumm bajommol duknozu (Public Sector

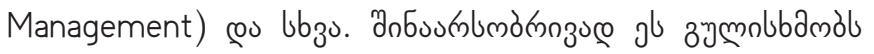

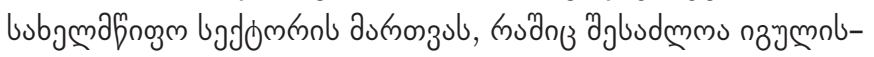

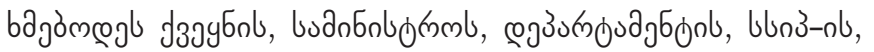

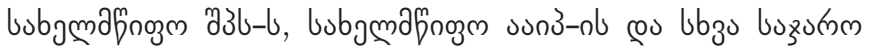

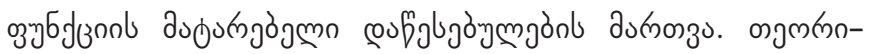

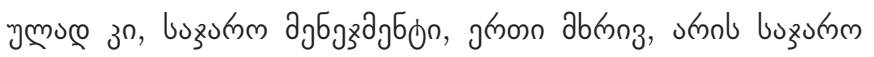

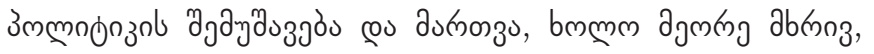

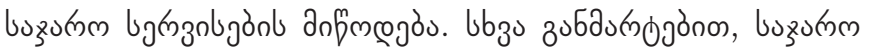

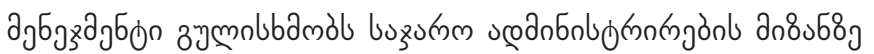

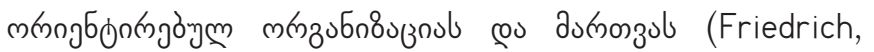

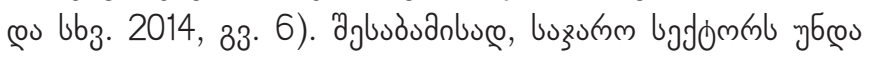

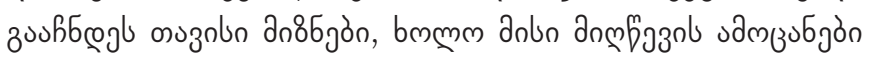
चб

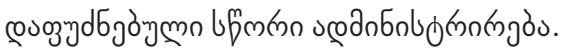

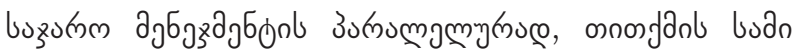

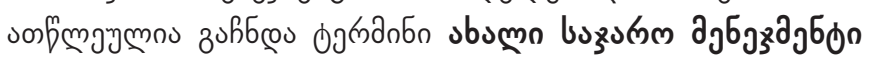

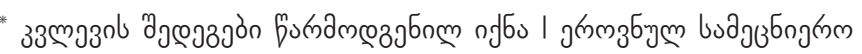

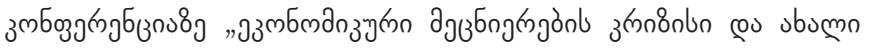
उsmuconzajònt dngosu"
}

(New Public Management - NPM), (nmagmbur zuhbno

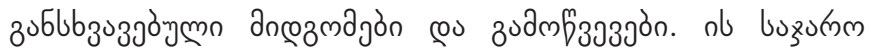

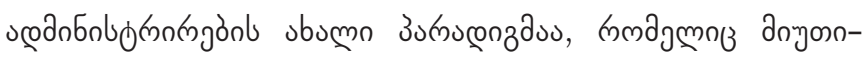

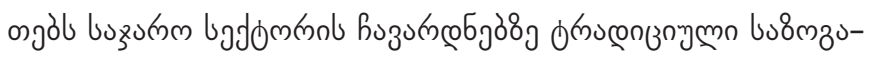

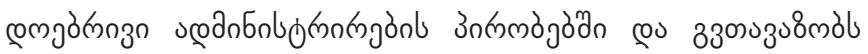

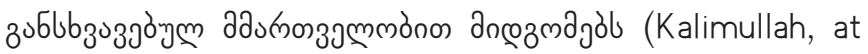
al.2012, 33. 1).

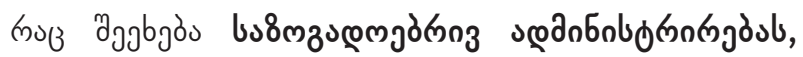

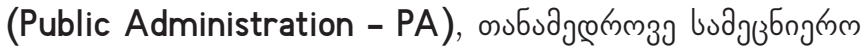

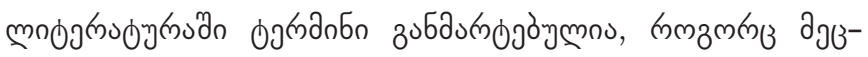

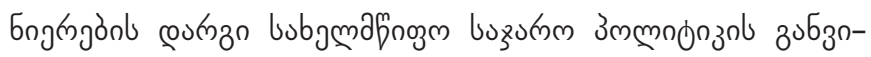

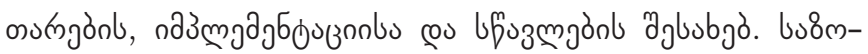

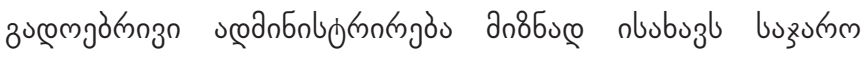

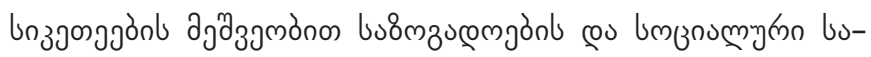

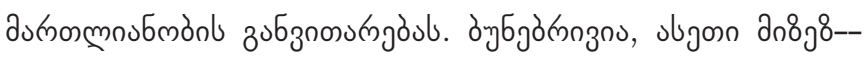

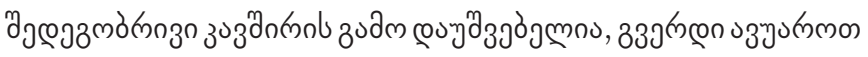

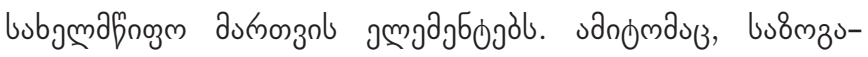

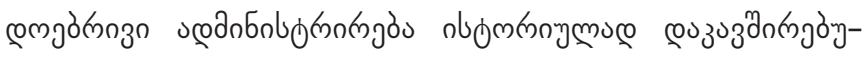

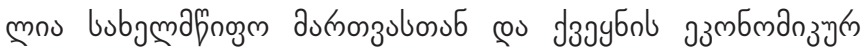

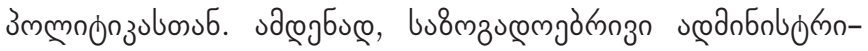

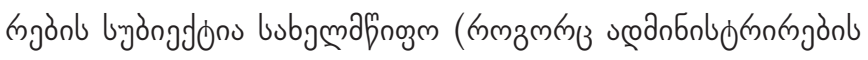

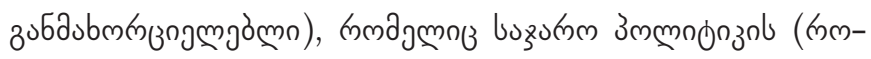

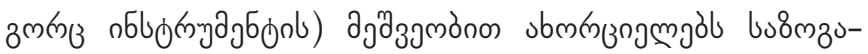

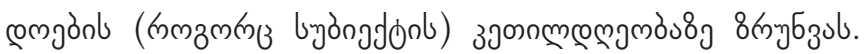

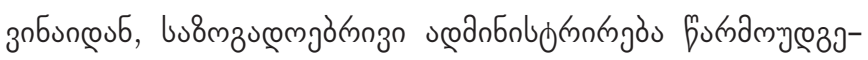

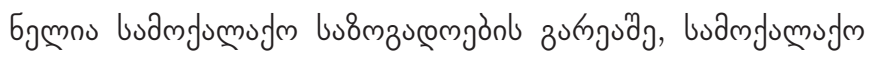

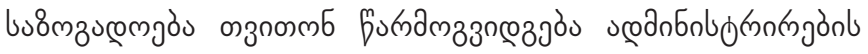

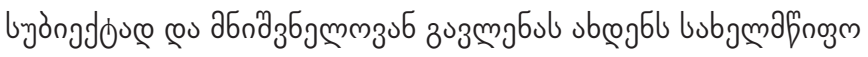

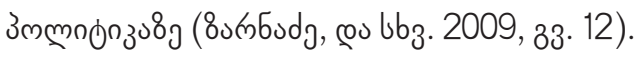

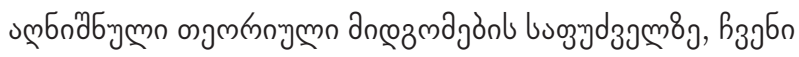

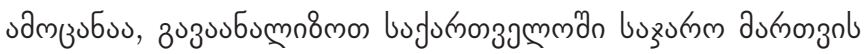

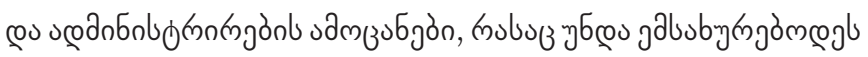

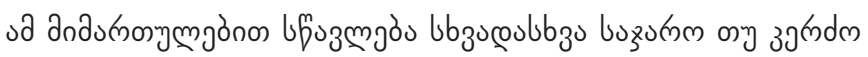

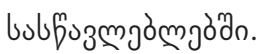




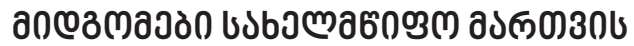

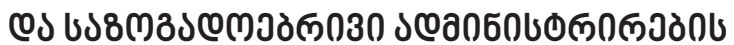

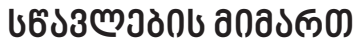

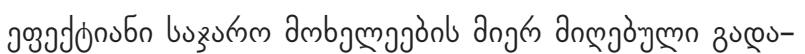
fysadom

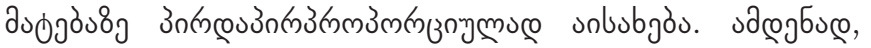

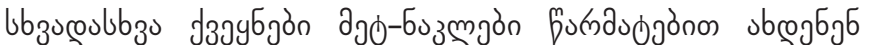

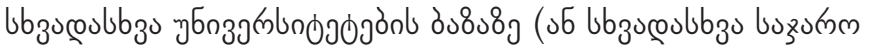

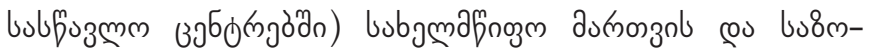

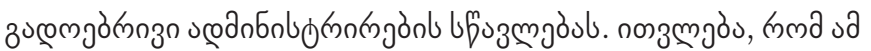

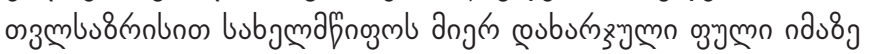

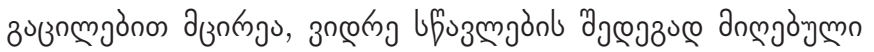

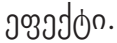

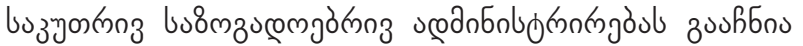

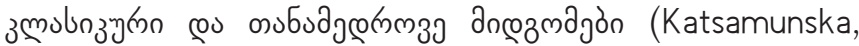

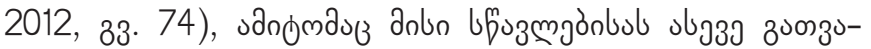

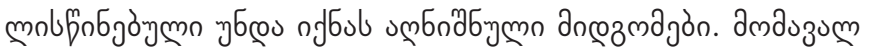

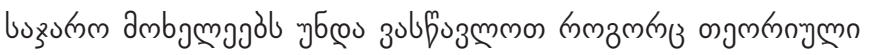

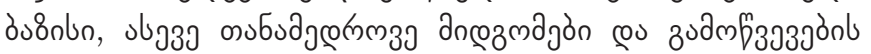

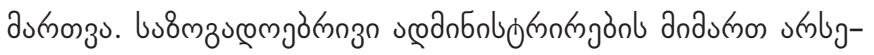

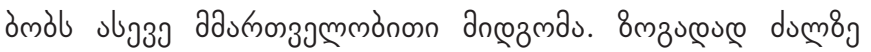

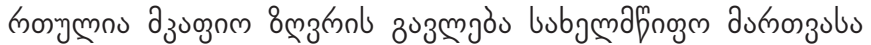

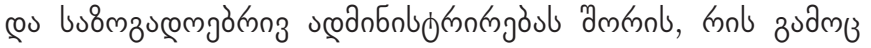

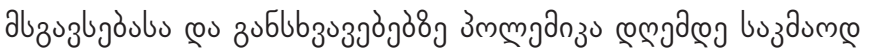
conono (Mora, at al. 2008, 33. 88).

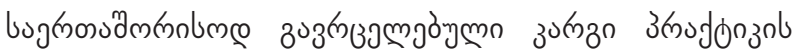

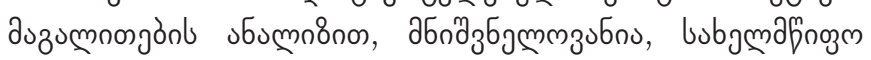

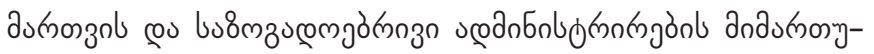

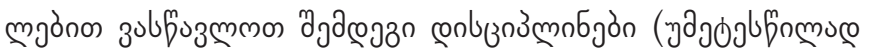

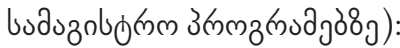

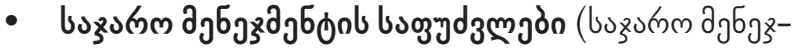

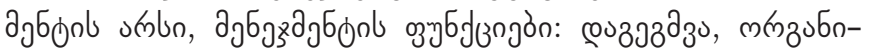

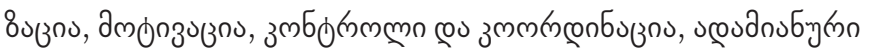

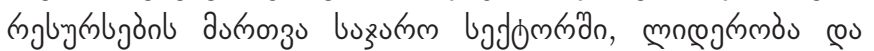

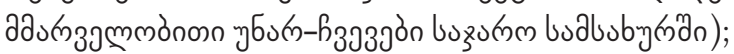

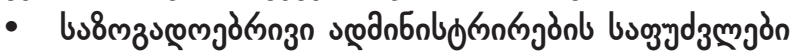

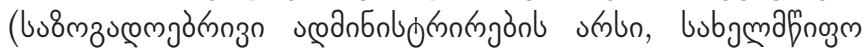

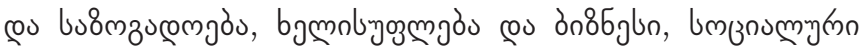

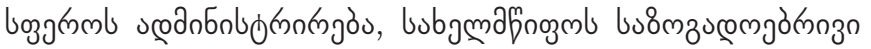

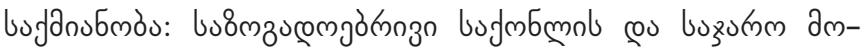

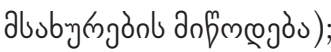

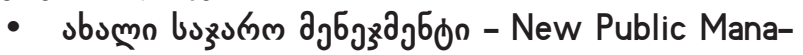

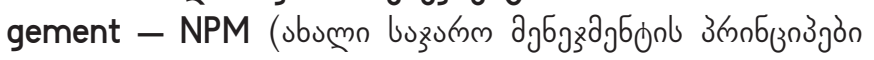

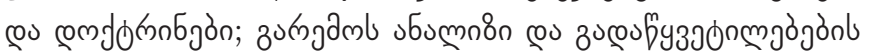

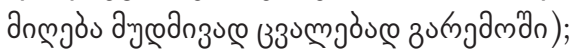

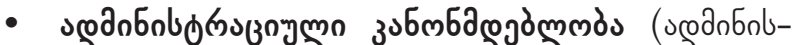

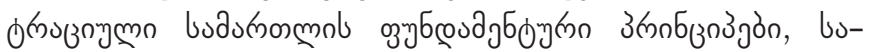

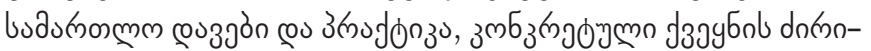

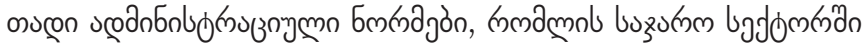

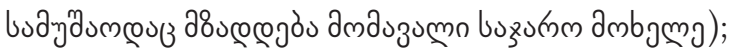

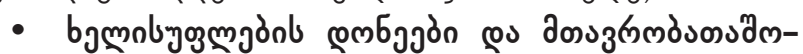

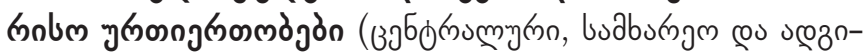

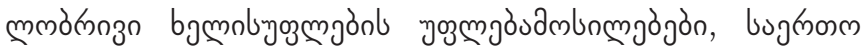

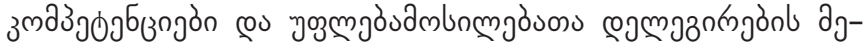

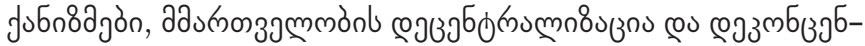
o(mo(zก) );

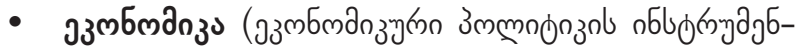

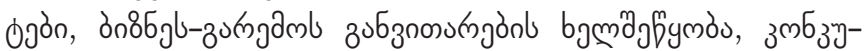

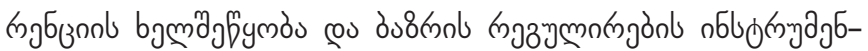

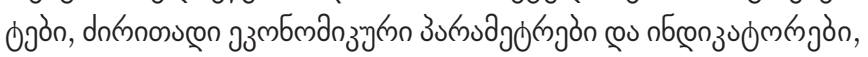

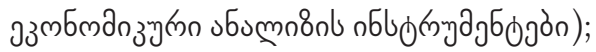

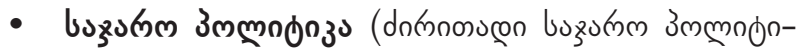

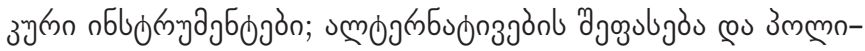

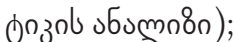

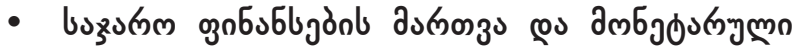

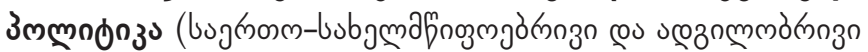

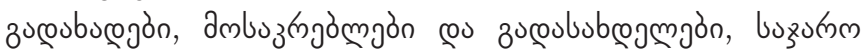

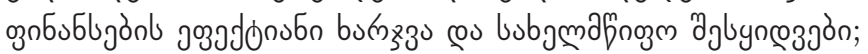

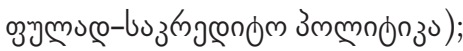

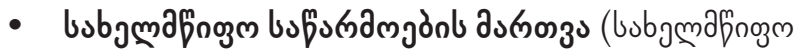

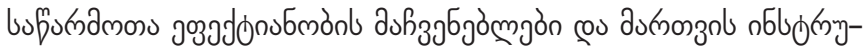
албоjòn agб

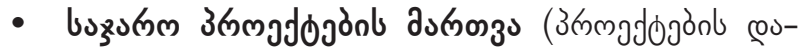

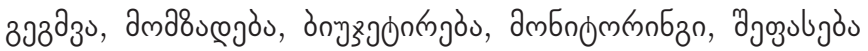
cou (nnluzjon bu asmonsu);

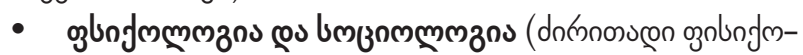

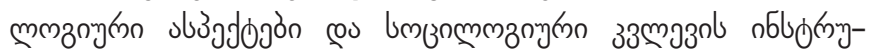
ajбogòn);

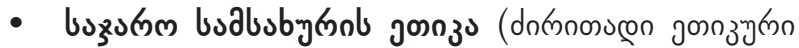

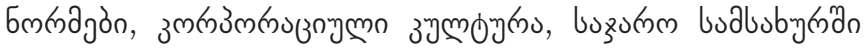

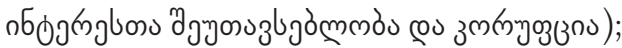

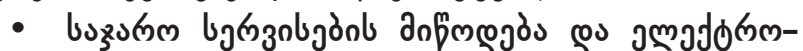

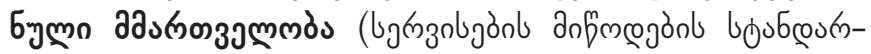

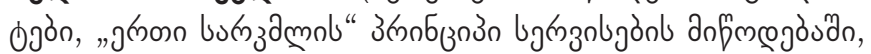

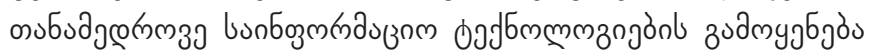

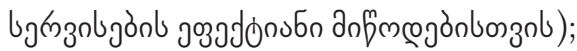

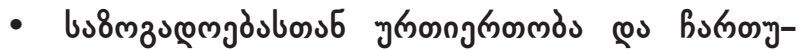

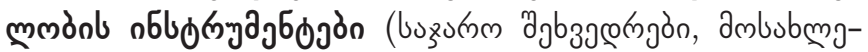

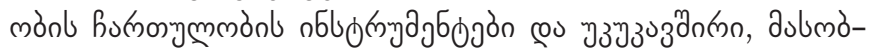

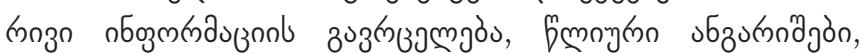

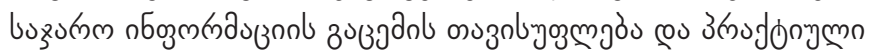
zodm $339 y 6 j \partial 0) ;$

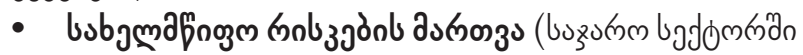
s

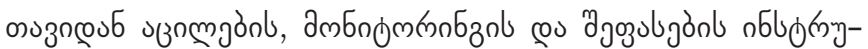

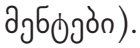




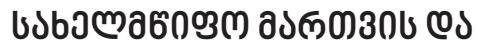

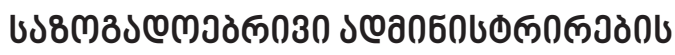

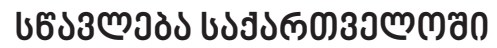

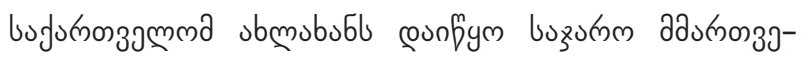

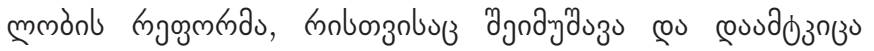

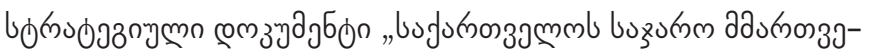

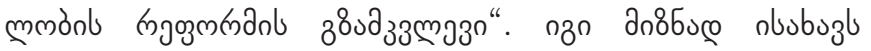
gмзмпи

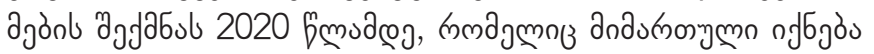

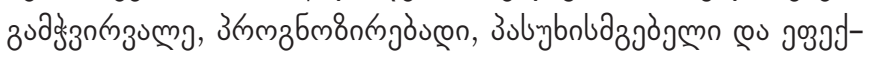

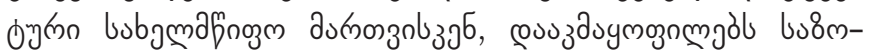

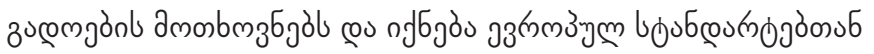

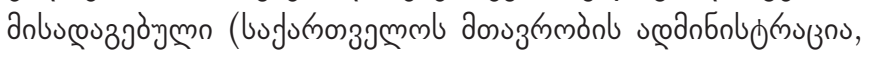
$2015,33.6)$.

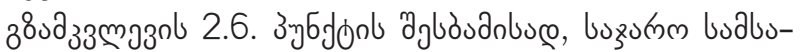

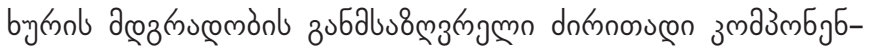

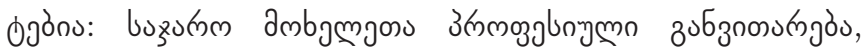

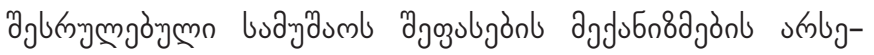

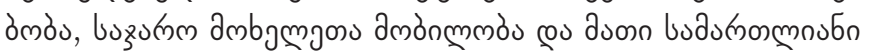

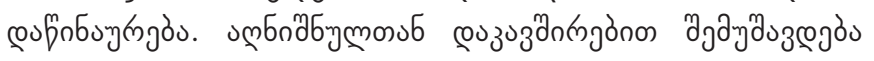

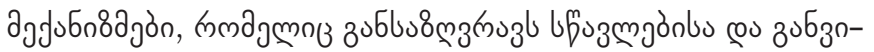

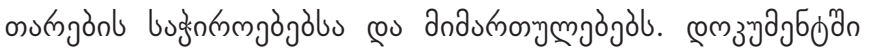

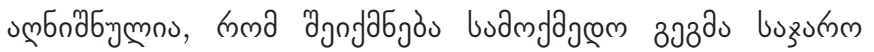

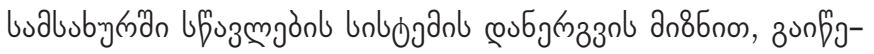

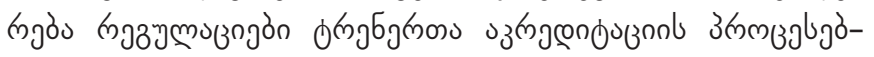

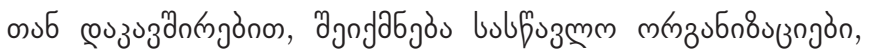

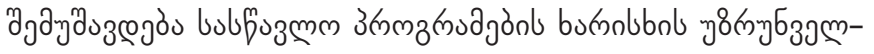

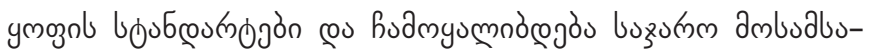

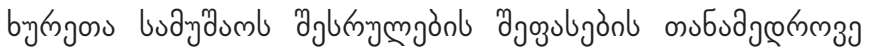
unbogas.

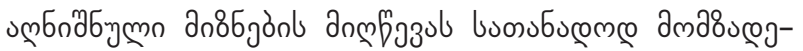

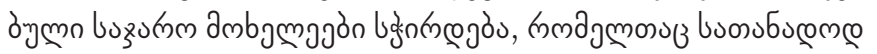
aلбjoson zus

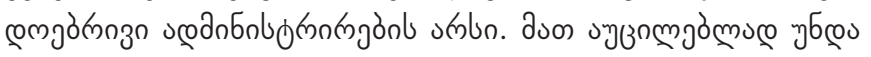

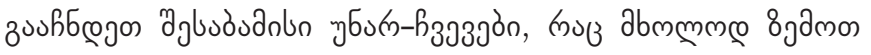

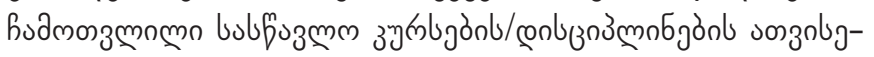
onnos ajbudmmo nf

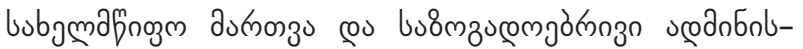

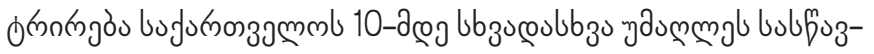

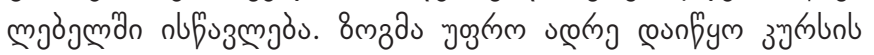

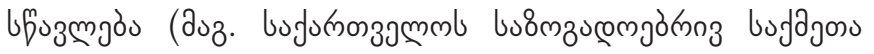

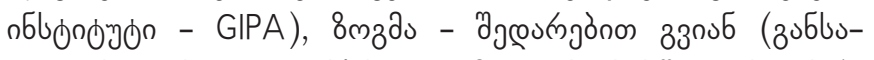

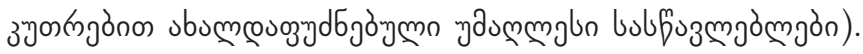

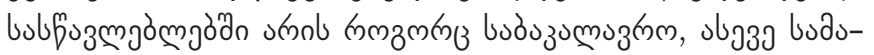

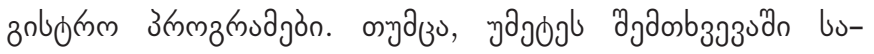

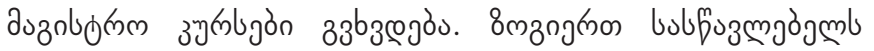

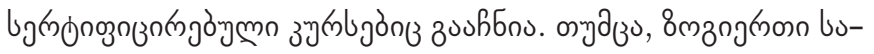

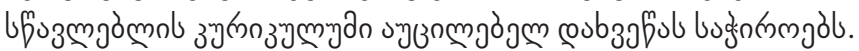

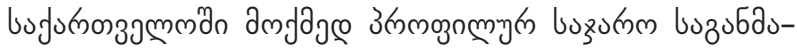

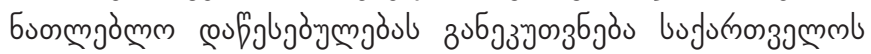

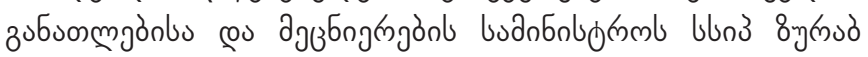

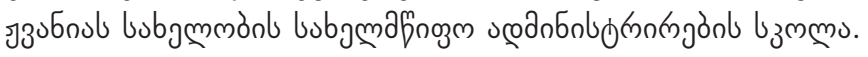

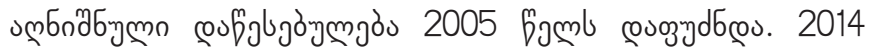

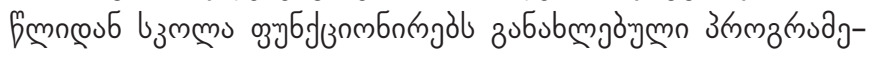

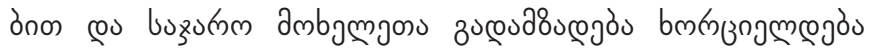

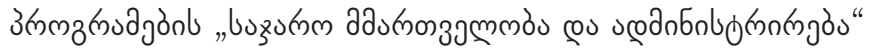

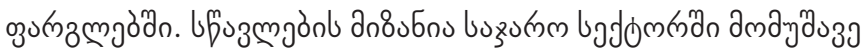

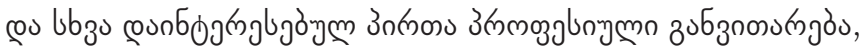

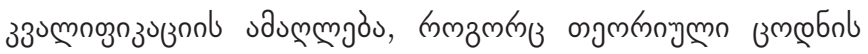

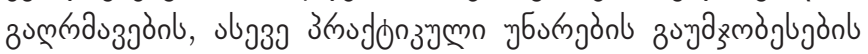

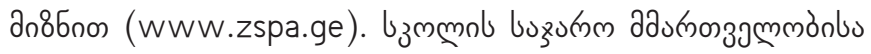

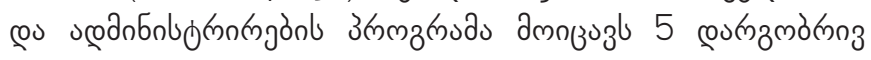
зचु⿰纟:

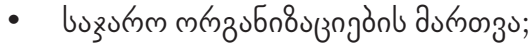

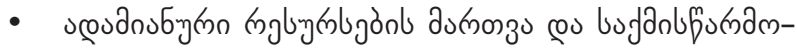
jò buzumm bajom mano;

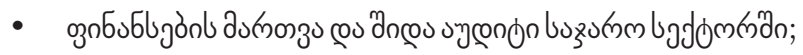

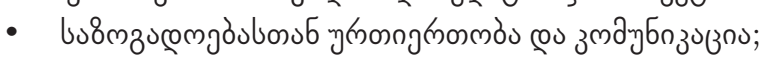

- nбognulob

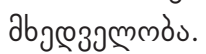

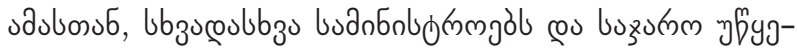

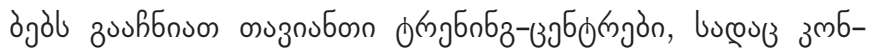

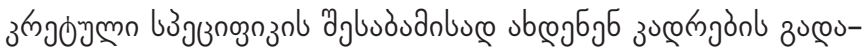

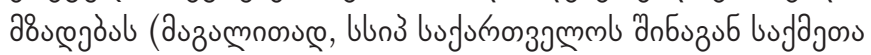

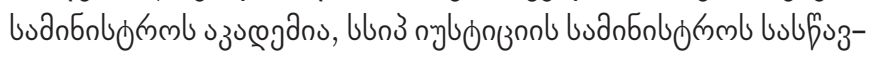

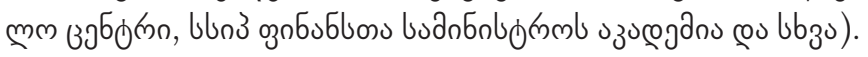

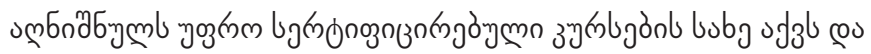

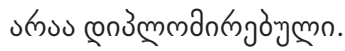

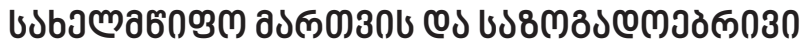

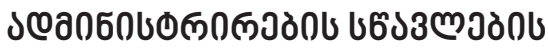

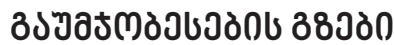

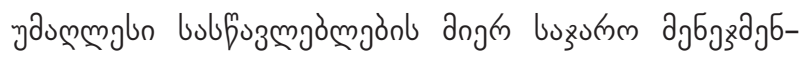

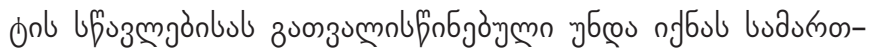

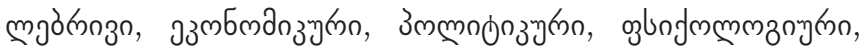

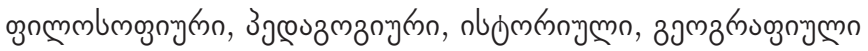

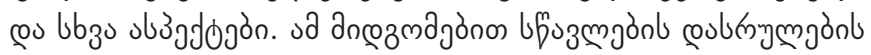

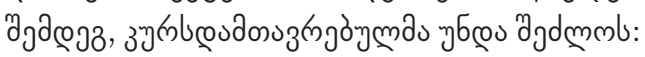

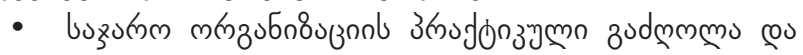
duknosso;

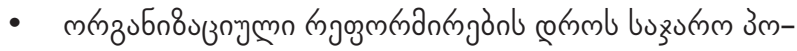

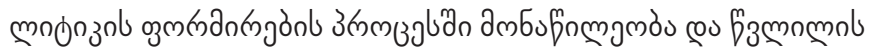
gुoubs;

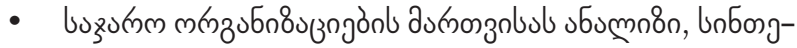

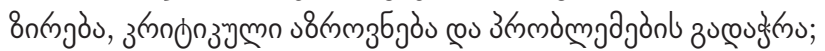




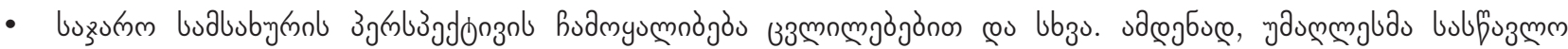
(Friedrich, @o bb3. 2014, 33. 93).

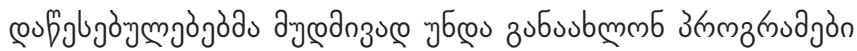

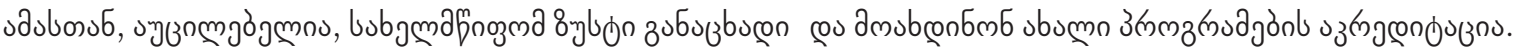

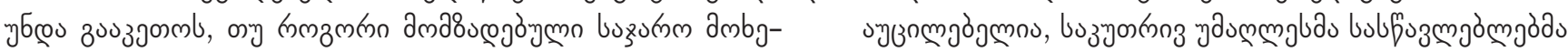

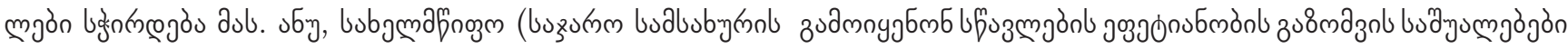

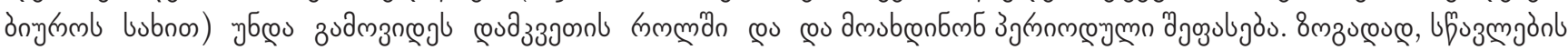

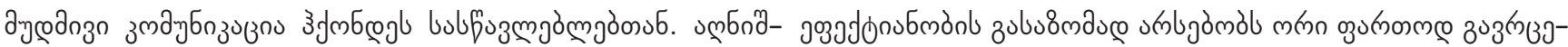

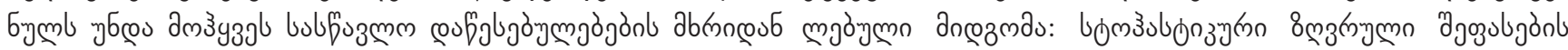

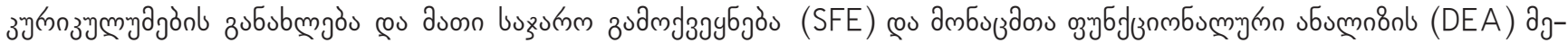

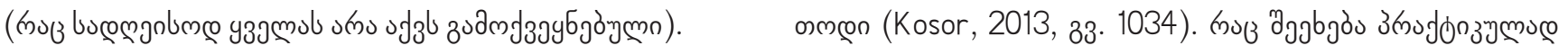

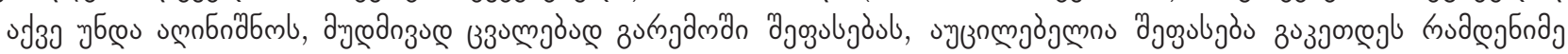

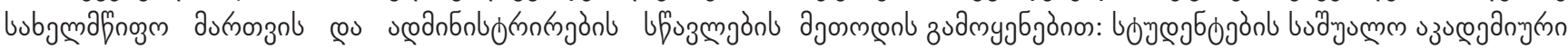

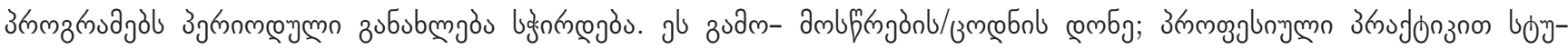

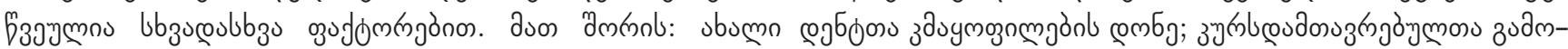

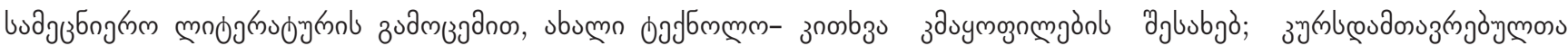

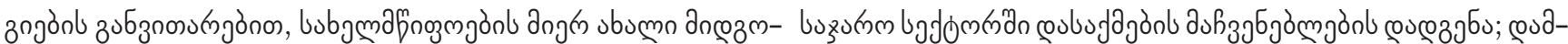

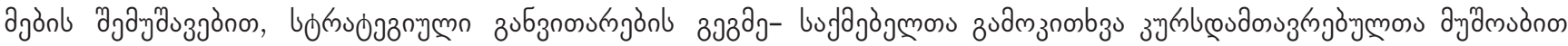

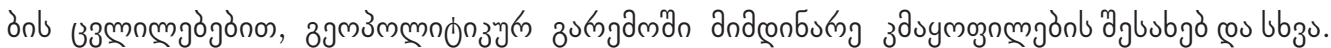

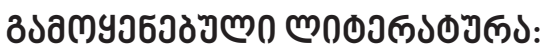

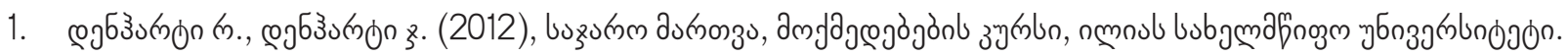

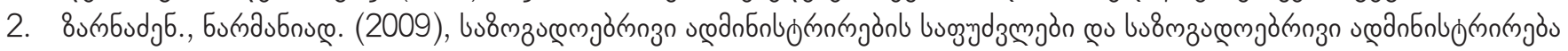

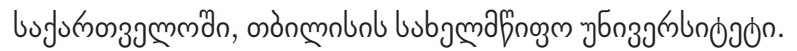

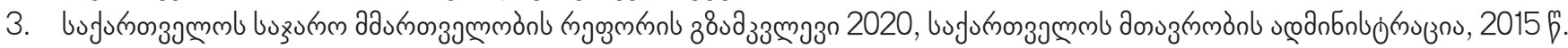

4. Betchoo N. (2016), Public Sector Management: a Millennial Insight, Bookboon.

5. Brans M., Coenen L. (2016), The Europeanization of Public Administration Teaching, Policy and Society, 35:4, pp. 333-349.

6. Friedrich P., Ukrainski K., Timpmann K. (2014), Teaching Public Management: Between Academic Disciplines, University of Tartu.

7. Hughs O. (2012), Public Management and Administration: An Introduction (4th Edition), Macmillan Palgrave, pp.74-102.

8. Kalimullah N, Ashraf Alam K., Ashaduzzaman Nour M. (2012), New Public Management: Emergence and Principles, BUP Journal, Vol.1, Issue 1, pp. 1-22.

9. Katsamunska P. (2012) Classical and Modern Approaches to Public Administration, Economic Alternatives, issue 1, pp. 74-81.

10. KosorM. (2013), Efficiency Measurement in Higher Education: Concepts, Methods and Perspective, Procedia - Social and Behavioral Sciences,\#106,pp. 1031 - 1038.

11. Mora C., Ticlau T. (2008), Managerial Approach to Public Administration, Transylvanian Review of Administrative Sciences, 24E, pp. 87-97.

12. Valdt G. (2014), Public Administration Teaching andInterdisciplinarity: Considering the Consequences, Teaching Public Administration, Vol. 32(2) pp. 169-193. 


\section{CURRENT MATTERS OF STATE MANAGEMENT AND PUBLIC ADMINISTRATION TEACHING IN GEORGIA}

\section{DAVIT NARMANIA}

https://doi.org/10.35945/gb.2018.06.009

Academic Doctor of Economics, Professor

Ivane Javakhishvili Tbilisi State University, Georgia

davit.narmania@tsu.ge

\section{KEYWORDS: MANAGEMENT, STATE MANAGEMENT, PUBLIC ADMINISTRATION, TEACHING}

\section{SUMMARY}

State Management and Public Administration Teaching is an actual matter of all civilized countries. Educated state officials who have profound knowledge in these subjects are more effective than public servants who have received the general education. Performance at work and decisions made by the effective public servants are directly reflected on country's success. Thus, different countries more or less successfully teach State Management and Public Administration at various universities (or different public training centers). It is considered that the money spent by the state is much less than the impact received from learning.

In this work we are trying to briefly overview the international experience and based on this reveal the effective teaching tools. Also, we present the possible ways to measure the efficiency of training. The work is focusing on the approaches and challenges of new public management, which require constant improvement in management. Therefore, it is necessary to update the teaching approaches to the State Management and Public Administration periodically.

In addition, the work is analyzing the current condition of state management and public administration teaching in Georgia. Based on the public sector reform progress and produced analysis the tasks for improving the teaching approaches is introducing. 